\title{
Prognostic Factors in Patients with COVID-19 Disease: A Retrospective Study in a Secondary Care Center
}

\author{
Clemens Stiegler*, Tanja Seel, Claus Schaefer \\ Email address: \\ Clemens.stiegler@klinikum.neumarkt.de (C. Stiegler) \\ ${ }^{*}$ Corresponding author
}

Department for Internal Medicine and Gastroenterology, Klinikum Neumarkt, Neumarkt in der Oberpfalz, Germany

To cite this article:

Clemens Stiegler, Tanja Seel, Claus Schaefer. Prognostic Factors in Patients with COVID-19 Disease: A Retrospective Study in a Secondary Care Center. Clinical Medicine Research. Vol. 10, No. 3, 2021, pp. 84-94. doi: 10.11648/j.cmr.20211003.14

Received: April 30, 2021; Accepted: May 19, 2021; Published: May 27, 2021

\begin{abstract}
The aim of this study was to identify clinical and laboratory characteristics with impact on outcome of patients with SARS-CoV2-Infection in a secondary care center in Germany. Therefore, a total of 69 hospitalized patients with COVID19, detected with positive Multiplex real-time PCR result, were recruited from March 2020 to May 2020 to investigate the influence of comorbidities, demographic information and laboratory parameters on outcome. Data of routine laboratory examinations of 57 patients were collected at admission to detect prognostic factors. Mean age of patients was 70.0 years (2199 years, median 74.0 years, SD 16,9). 28 patients $(40,6 \%)$ had a severe course of disease (death and/or need for intensive care medicine), 20 patients (29\%) died. LDH $>460 \mathrm{U} / 1$ ( $\mathrm{p}=0.004$, OR 12.99, 95\% CI 2.23-75.67), Diabetes mellitus ( $\mathrm{p}=0.021$, OR 9.53, 95\% CI 1.14-64.48) and Troponin T $>38 \mathrm{pg} / \mathrm{ml}(\mathrm{p}=0.026$, OR 6.04, 95\% CI 1.24-29.43) were associated with occurrence of severe illness in multivariate analysis. Elevated Troponin $\mathrm{T}>38 \mathrm{pg} / \mathrm{ml}(\mathrm{p}=0.002$, HR 8.22, 95\% CI $2.19-30.88)$ and Diabetes mellitus ( $\mathrm{p}=0.05$, HR 3.14, 95\% CI $1-9.85$ ) were also associated with death. Patients with these conditions should be monitored closely.
\end{abstract}

Keywords: COVID-19, Risk Factors, Severe Disease, Diabetes Mellitus, Troponin T, LDH, Laboratory Parameters

\section{Introduction}

In March 2020, COVID-19 pandemic spread around the world with great impact on health care systems in all countries. After a decline of incidences in the summer, a second wave of infection began in autumn with higher number of cases in Germany than before. Even though vaccination is available by now, the pandemic is still not averted and will persist for several months. Unlike other entities of virus pneumonies, the disease course varies strongly with some younger patients without comorbidities developing ARDS and SIRS as well as older patients. Furthermore, the diversity of symptoms is great. Besides cough, shortness of breath, fever, GI-symptoms like diarrhea or abdominal pain, other major events in patients with COVID-19 are arterial or venous thromboembolism, neurological symptoms or kidney disease, which may be the result of endothelial damage, caused by the virus. [36] Studies of risk factors for severe disease particularly came from China, Wuhan. Therefore, external validity of data for other countries may be limited. Older age, hypertension, high cytokine levels (IL-2R, IL-6, IL-10, and TNF- $\alpha$ ), and high lactate dehydrogenase level were significantly associated with severe COVID-19 on admission [1]. An analysis by the joint WHO-China fact-finding mission found that patients older than 60 years and those with comorbidities had the highest risk for severe disease and death [2]. The case fatality rate (CFR) in patients without comorbidities was $1.4 \%$, whereas it was between $7.6 \%$ to $13.2 \%$ for patients with comorbidities such as cardiovascular disease, diabetes, hypertension, chronic respiratory disease and patients with cancer [3]. Clinical prediction tools for pneumonia such as CRB-65 are developed for bacterial infections but remain not applicable in viral infections $[4,5]$. To our knowledge, studies in secondary care centers with different patient collectives compared with patients of tertiary care centers do not exist and there are no simple prediction rules for stratifying patients. Hence, the aim of this study was to investigate risk factors in a patient collective of a secondary care center to 
stratify patients into "high risk" and "low risk".

\section{Materials and Methods}

\subsection{Study Design}

A retrospective single-center observational study was conducted from March 2020 to May 2020 in Neumarkt city hospital, Bavaria, Germany. The study was approved by the responsible ethics committee.

We included all hospitalized patients with age $>18$ years and positive result of real-time reverse-transcription polymerase chain reaction (RT-PCR) for detection of Severe acute respiratory syndrome coronavirus type 2 (SARSCoV2). 69 patients had a positive PCR test in aforementioned time period. 12 patients were hospitalized because of other reasons and got infected due to the hospital stay, so they were excluded from analysis of laboratory data at admission. Laboratory results of 57 patients were investigated.

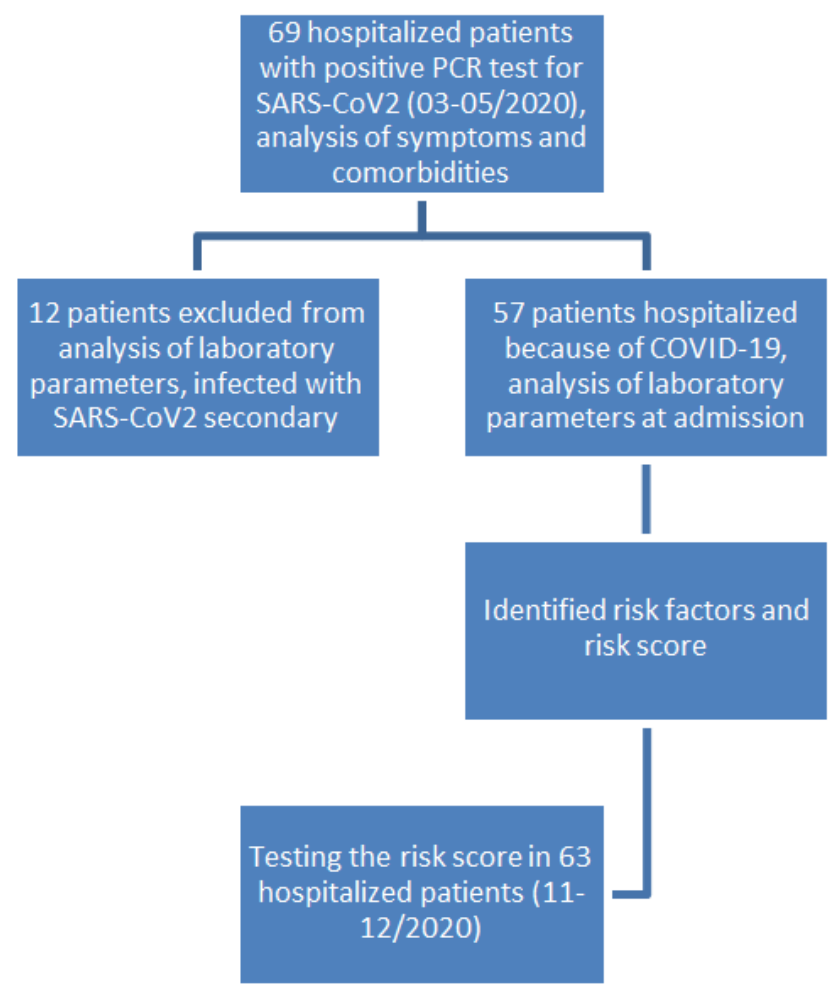

Figure 1. Study flow chart.

\subsection{Data Collection}

Nasal swabs were used for confirming COVID-19 with RT-PCR using the Cellgene system. Data were collected on admission including demographic information, comorbidities, symptoms, laboratory examinations and CT scanning of chest, when carried out. Length of stay and outcome of each patient were recorded. Patients were followed up by phone call after hospital discharge. Overall mortality was defined as mortality within 60 days after hospital admission.

\subsection{Statistical Methods}

Patients with COVID-19 were divided into high risk and low risk group depending on course of disease. Disease course with need for intensive care medicine and/or death within 60 days after hospital admission was classified as "severe". Means with standard deviation (SD) were used to characterize the patient sample. Univariate analysis was initially used to compare risk factors separately. T-test was used for comparing continuous variables while $\chi^{2}$ or Fisher's exact test were used for categorical data analysis, as appropriate. Cut-off points were identified using Youden's index of receiver operator characteristic (ROC) and clinical relevant cut-off levels respectively. The clinical and laboratory variables with significant association with severe disease and phi-values $>0.36$ as marker of effect size in univariate analysis were retained for multivariate testing using a logistic regression model. The clinical and laboratory variables with significant association with death in univariate analysis and $\mathrm{phi} \geq 0.3$ were included in multivariate analysis against overall mortality by a cox regression model with backward elimination procedure (conditional likelihood ratio test and elimination, if $p \geq 0.1$ ). Score points for each predictor for severe disease were assigned relative to the regression coefficient. The cut-off point of the total score for the distinction between low risk and high risk was identified following Youden's index of receiver operator characteristic (ROC). Sensitivity, specifity and the area under the receiver operator characteristic (AUROC) were calculated (training set). The risk score was evaluated in a testing set, for which 63 hospitalized patients with detected COVID-19 were recruited from November, 1, 2020 to December, 15, 2020. Sensitivity, specifity and the area under the receiver operator characteristic (AUROC) of the score were calculated in the testing set. Survival analysis was performed using KaplanMeier analysis and log-rank-test between low risk and high risk group. Further Kaplan-Meier analyses was performed for testing each of the score variables to show impact on survival. All tests were two sided and a $p$-value $\leq 0.05$ was considered significant. Statistical analysis was performed using SPSS version 26.

\section{Results}

\subsection{Patient Characteristics}

Table 1 shows baseline characteristics of the different patient groups. $55.1 \%$ of patients were male. Mean age was 70.0 years (21-99 years, median 74.0 years, SD 16,9), $72.5 \%$ of patients were older than 65 years. Figure 1 shows distribution of age. $15.9 \%$ of patients were hospitalized because of other causes and infected with SARS-CoV2 secondary. 52 patients $(75.4 \%)$ had comorbidities with hypertension as the most commonly observed comorbidity (68.1\%), followed by chronic kidney disease (33.3\%), atrial fibrillation (27.5\%) and diabetes mellitus (21.7\%). 86.7\% $(13 / 15)$ of the diabetics had diabetes type 2, one patient type 1 and another patient type $3 \mathrm{e}$ ( $6.7 \%$ in each case). $15.4 \%$ of 
patients with diabetes type 2 had a therapy with metformin. $46.7 \%$ of all diabetics were treated with insulin.

$15.3 \%$ of patients had pulmonary disease. Respiratory symptoms (dyspnea and cough, 79.7\%) and fever (55.1\%) were most common, whereas GI-symptoms such as diarrhea were observed in $33.3 \%$. 20.3\% had smoking history. 28 patients $(40.6 \%)$ had a severe disease course with 16 patients
(23.2\%) being admitted to intensive care unit (ICU). 20 patients $(29 \%)$ died during hospital stay. The mean length of stay was 22.9 days (range 100 days, SD 18.7 days). The mean survival time of patients, who died, was 10.4 days (SD 8) after admission. Symptoms persisted at time of hospital admission since 5.8 days (SD 4.6) on average.

Table 1. Population description and comparison between survivors/patients without need of intensive care medicine and patients who died within 60 days after admission/ patients with severe disease course (admission to ICU and/or death).

\begin{tabular}{|c|c|c|c|c|c|}
\hline & Total population, $n=69$ & Survivors, $n=49$ /Non severe, $n=42$ & Severe disease, $\mathbf{n}=\mathbf{2 7}$ & Deceased, $n=20$ & p-value; $\phi$ \\
\hline \multicolumn{6}{|l|}{ Social-demographic details } \\
\hline \multirow{2}{*}{ Age } & \multirow{2}{*}{$70 \pm 16.9$} & $66 \pm 17.4$ & & \multirow[t]{2}{*}{$79,9 \pm 10.8$} & $<0.001$ \\
\hline & & $67.38 \pm 17.48$ & $74.11 \pm 15.51$ & & 0.108 \\
\hline \multirow{2}{*}{ Age $>65$ years* } & \multirow{2}{*}{$45(65.2)$} & $27(55.1)$ & & \multirow[t]{2}{*}{$18(90)$} & $0.006 ; 0.332$ \\
\hline & & $26(61.9)$ & $19(70.4)$ & & 0.471 \\
\hline \multirow{2}{*}{ Male } & \multirow{2}{*}{$38(55.1)$} & $27(55.1)$ & & \multirow[t]{2}{*}{$11(55)$} & 0.994 \\
\hline & & $22(52.4)$ & $16(59.3)$ & & 0.575 \\
\hline \multirow{2}{*}{ Smoking history } & \multirow{2}{*}{$14(20.3)$} & $9(18.4)$ & & \multirow[t]{2}{*}{$5(25)$} & 0.534 \\
\hline & & $6(14.3)$ & $8(29.6)$ & & 0.122 \\
\hline \multicolumn{6}{|l|}{ Clinical features } \\
\hline \multirow{2}{*}{ Fever } & \multirow{2}{*}{$38(55.1)$} & $26(53.1)$ & & \multirow[t]{2}{*}{$12(60)$} & 0.599 \\
\hline & & $23(54.8)$ & $15(55.6)$ & & 0.948 \\
\hline \multirow{2}{*}{$\begin{array}{l}\text { Lack of respiratory } \\
\text { symptoms }\end{array}$} & \multirow{2}{*}{$14(20.3)$} & $12(24.5)$ & & \multirow[t]{2}{*}{$2(10)$} & 0.322 \\
\hline & & $12(28.6)$ & $2(7.4)$ & & $0.033 ; 0.257$ \\
\hline \multirow{2}{*}{ GI symptoms } & \multirow{2}{*}{$23(33.3)$} & $16(32.7)$ & & $7(35)$ & 0.895 \\
\hline & & $15(35.7)$ & $8(29.6)$ & & 0.553 \\
\hline & & $3(6.1)$ & & $2(10)$ & 0.627 \\
\hline Neurological symptoms & $5(7.2)$ & $3(7.1)$ & $2(7.4)$ & & 1.000 \\
\hline Bilateral pulmonary infiltrates & $31(449)$ & $21(42.9)$ & & $10(50)$ & 0.255 \\
\hline in CT scan of chest & $31(44.9)$ & $15(35.7)$ & $16(59.3)$ & & 0.476 \\
\hline $\mathrm{SpO}_{2}<95 \%$ without $\mathrm{O}_{2}-$ & & $26(53.1)$ & & $11(55)$ & 0.346 \\
\hline supplementation ${ }^{\circ}$ & $37(53.6)$ & $19(45.2)$ & $18(66.7)$ & & $0.017 ; 0,301$ \\
\hline & & $22(44.9)$ & & $13(65)$ & 0.130 \\
\hline ACE inhibitors/Sartans & $35(50.7)$ & $20(47.6)$ & $15(55.6)$ & & 0.520 \\
\hline & & $7(14.3)$ & & $8(40)$ & 0.027 \\
\hline ICU & $15(21.7)$ & 0 & $15(55.6)$ & & - \\
\hline Comorbidities & & & & & \\
\hline & & $34(69.4)$ & & $18(90)$ & 0.122 \\
\hline Overall $^{\circ}$ & $52(75.4)$ & $31(73.8)$ & $21(77.8)$ & & 0.709 \\
\hline & & $5(10.2)$ & & $4(20)$ & 0.431 \\
\hline Immunsuppressive therapy & $9(13.0)$ & $5(11.9)$ & $4(14.8)$ & & 0.729 \\
\hline & & $31(63.3)$ & & $16(80)$ & 0.176 \\
\hline Hypertension & $47(68.1)$ & $28(66.7)$ & $19(70.4)$ & & 0.747 \\
\hline Chronic kidney disease ${ }^{\circ *}$ & & $10(20.4)$ & & $13(65)$ & $<0.001 ; 0.429$ \\
\hline (stage $\geq 3$ ) & $23(33.3)$ & $8(19)$ & $15(55.6)$ & & $0.002 ; 0.378$ \\
\hline & & $8(16.3)$ & & $5(25)$ & 0.500 \\
\hline Chronic heart failure & $13(18.8)$ & $7(16.7)$ & $6(22.2)$ & & 0.565 \\
\hline Peripheral artery occlusive & $4(58)$ & $1(2)$ & & $3(15)$ & 0.070 \\
\hline disease & $4(5.8)$ & 0 & $4(14.8)$ & & $0.020 ; 0.309$ \\
\hline & & $5(10.2)$ & & $5(25)$ & 0.140 \\
\hline Coronary heart disease & $10(14.5)$ & $5(11.9)$ & $5(18.5)$ & & 0.497 \\
\hline & & $14(28.6)$ & & $5(25)$ & 0.763 \\
\hline Atrial fibrillation & $19(27.5)$ & $14(33.3)$ & $5(18.5)$ & & 0.179 \\
\hline Diabetes mellitus $^{\circ *}$ & $15(217)$ & $6(12.2)$ & & $9(45)$ & $0.008 ; 0.360$ \\
\hline (all types) & $15(21.7)$ & $4(9.5)$ & $11(40.7)$ & & $0.002 ; 0.369$ \\
\hline & & $5(10.2)$ & & $6(30)$ & 0.067 \\
\hline Pulmonary disease & $11(15.9)$ & $3(7.1)$ & $8(29.6)$ & & 0,$019 ; 0.300$ \\
\hline
\end{tabular}

Continuous parameters presented as mean $\pm \mathrm{SD}$, categorical data as $\mathrm{n}(\%)$. All clinical data were collected on admission. p-value refers to the comparison of patients with mild disease/survival group and patients with severe disease/death group. p-value $\leq 0.05$ represents significant difference between the groups. $\phi=$ effect size. ${ }^{\circ}$ variables cited above were included in multivariate analysis against the endpoint "severe disease". * variables cited above were included in the cox regression model against the endpoint death. ICU= intensive care unit. 


\section{Frequeny}

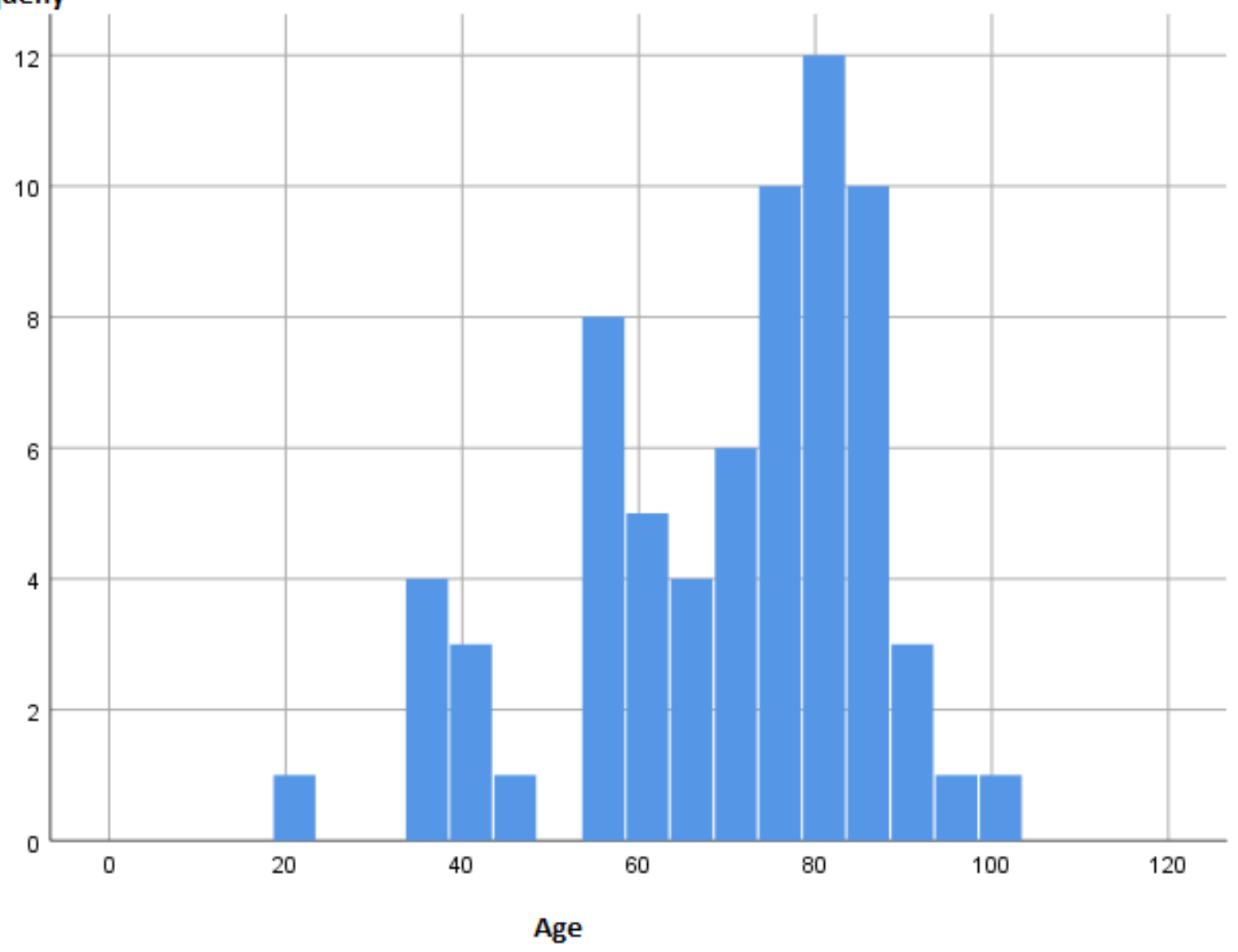

Figure 2. Distribution of age.

Table 2. Comparison of laboratory parameters of survivors and non-survivors and of patients with mild disease and patients with severe disease.

\begin{tabular}{|c|c|c|c|}
\hline Parameter & Survivors / Non severe disease & Overall death / Severe disease & p-value \\
\hline \multirow{2}{*}{ Lymphocytes } & $0.94 \pm 0.521$ & $1.11 \pm 0.921$ & 0.529 \\
\hline & $0.95 \pm 0.55$ & $1.05 \pm 0.80$ & 0.565 \\
\hline \multirow{2}{*}{ Hemoglobin [g/dl] } & $13.23 \pm 2,25$ & $11.79 \pm 2,6$ & 0.041 \\
\hline & $13.28 \pm 2.31$ & $12.11 \pm 2.48$ & 0.073 \\
\hline \multirow{2}{*}{ Serum sodium $[\mathrm{mmol} / \mathrm{l}]$} & $136.74 \pm 4.42$ & $138,38 \pm 8.91$ & 0.490 \\
\hline & $138.18 \pm 7.66$ & $138.04 \pm 7.51$ & 0.312 \\
\hline Serum potassium $[\mathrm{mmol} / \mathrm{l}]$ & $4.23 \pm 0,54$ & $4.75 \pm 0,89$ & 0.007 \\
\hline \multirow{2}{*}{ Lactate $[\mathrm{mg} / \mathrm{dl}]$} & $18.19 \pm 5,64$ & $27.63 \pm 14,22$ & 0,045 \\
\hline & $18.48 \pm 5,87$ & $24.81 \pm 13,30$ & 0.089 \\
\hline \multirow{2}{*}{ Creatine kinase [U/l] } & $410.74 \pm 872,03$ & $604.00 \pm 1338,14$ & 0.518 \\
\hline & $399.62 \pm 919,08$ & $570.00 \pm 1162,41$ & 0.536 \\
\hline Creatinine $[\mathrm{mg} / \mathrm{dl}]$ & $1.40 \pm 1,83$ & $2.36 \pm 1,83$ & 0.080 \\
\hline \multirow{2}{*}{ C-reactive protein $[\mathrm{mg} / \mathrm{l}]$} & $68.76 \pm 66,37$ & $99.73 \pm 93,07$ & 0.160 \\
\hline & $53.09 \pm 47,09$ & $117.64 \pm 94,82$ & 0.006 \\
\hline \multirow{2}{*}{ D-Dimere $[\mathrm{mg} / \mathrm{l}]$} & $1.85 \pm 3,16$ & $4.26 \pm 6,75$ & 0.219 \\
\hline & $1.20 \pm 1,01$ & $4.61 \pm 6,69$ & 0.035 \\
\hline \multirow{2}{*}{ Lactate dehydrogenase [U/1] } & $379.28 \pm 198,60$ & $449.13 \pm 184,85$ & 0.226 \\
\hline & $339.46 \pm 115,63$ & $497.05 \pm 258,39$ & 0.012 \\
\hline \multirow{2}{*}{ Alanine aminotransferase [U/1] } & $46.53 \pm 45,35$ & $37.13 \pm 52,85$ & 0.501 \\
\hline & $42.27 \pm 43,65$ & $46.86 \pm 53,64$ & 0.721 \\
\hline \multirow{2}{*}{ Troponin T [pg/ml] } & $29.58 \pm 42,92$ & $143.18 \pm 172,09$ & 0.036 \\
\hline & $31.00 \pm 45,14$ & $113.68 \pm 158,89$ & 0.050 \\
\hline
\end{tabular}

The bolded values are $p$-values $\leq 0.05$, indicating significant difference between the subgroups in t-test 


\subsection{Risk Factors of Severe Disease and Mortality}

Patients with severe disease had significant higher values of serum potassium $(\mathrm{p}=0.007)$, creatinine $(\mathrm{p}=0.043)$, Creactive protein $(\mathrm{p}=0.035), \mathrm{D}$-dimere $(\mathrm{p}=0.035)$ and lactate dehydrogenase $(\mathrm{p}=0.012)$.

The subgroup of patients, who died, had significant lower levels of hemoglobin $(\mathrm{p}=0.41)$ and higher levels of lactate $(\mathrm{P}=0.045)$ and Troponin $\mathrm{T}(=.036)$. Table 2 shows the comparison of laboratory parameters between the subgroups.

After defining cut off points using ROC-analysis or clinical relevant cut off values, univariate analysis with $\chi^{2}$ or Fisher's exact test was performed to compare the different subgroups, as shown in table 3 .

Table 3. Univariate association analysis of categorized laboratory parameters and severe disease or death.

\begin{tabular}{lll}
\hline \multirow{2}{*}{ Parameter } & p-value; $\phi$ & \\
\cline { 2 - 3 } & Severe & Death \\
\hline Serum potassium $>4.7 \mathrm{mmol} / \mathrm{l}^{*}$ & $0.041 ; 0.307$ & $0.011 ; 0.355$ \\
$\Delta \mathrm{Na}^{+}>6.5 \mathrm{mmol} / 1$ & 0.070 & 0.205 \\
$\mathrm{CRP}>49 \mathrm{mg} / \mathrm{dl}{ }^{\circ}$ & $0.017 ; 0.380$ & 0.312 \\
$\mathrm{LDH}>460 \mathrm{U} / 1^{\circ *}$ & $<0.001 ; 0.476$ & $0.023 ; 0.314$ \\
$\mathrm{D}-\mathrm{Dimere}>1.5$ & $0.009 ; 0.358$ & 0.270 \\
Hemoglobine $<12 \mathrm{~g} / \mathrm{dl}$ & 0.114 & 0.050 \\
Lactate $>22 \mathrm{mmol} / 1$ & 0.287 & 0.052 \\
Troponin $\mathrm{T}>38 \mathrm{pg} / \mathrm{ml} l^{\circ *}$ & $0.004 ; 0.413$ & $<0.001 ; 0.571$ \\
\hline
\end{tabular}

The bolded $\mathrm{p}$-values are significant $(\mathrm{p} \leq 0.05)$. ${ }^{\circ}$ variables cited above were included in multivariate analysis against the endpoint "severe disease". * variables cited above were included in the cox regression model against the endpoint death. $\mathrm{ICU}=$ intensive care unit.

Table 4. Multivariate, logistic regression model associated with severe course of disease $(p<0.001)$.

\begin{tabular}{lllll}
\hline Parameter & p-value & $\begin{array}{l}\text { regression } \\
\text { coefficient } \mathbf{B}\end{array}$ & Odds $(\mathbf{9 5 \%}$ CI) & Score \\
\hline LDH $>460 \mathrm{U} / 1$ & 0,004 & 2.564 & $12.99(2.23-75.67)$ & 3 \\
$\mathrm{DM}$ & 0,021 & 2.254 & $9.53(1.41-64.48)$ & 2 \\
Trop T & 0.026 & 1.798 & $6.04(1.24-29.43)$ & 1 \\
\hline
\end{tabular}

Table 5. Risk-score, sensitivity and specifity, training set.

\begin{tabular}{lllll}
\hline Total score & $\mathbf{n}$ & Severe disease (\%) & Sensitivity & Specifity \\
\hline 0 & 23 & $2(8.7)$ & 1.0 & 0 \\
1 & 11 & $3(27.3)$ & 0.909 & 0.568 \\
2 & 4 & $1(25)$ & 0.773 & 0.784 \\
3 & 12 & $8(66.7)$ & 0.727 & 0.865 \\
4 & 5 & $4(80)$ & 0.364 & 0.973 \\
5 & 0 & - & - & - \\
6 & 4 & $4(100)$ & .182 & 1.0 \\
\hline
\end{tabular}

The boulded value represents the cut off level following Youden's index of receiver operator curve.

The following categorical variables were entered in a stepwise logistic regression analysis against severe disease: LDH $>460 \mathrm{U} / \mathrm{l}$, Troponin $\mathrm{T}>38 \mathrm{pg} / \mathrm{ml}, \quad \mathrm{CRP}>49 \mathrm{mg} / \mathrm{dl}$, chronic kidney disease (stage $>3$ ), diabetes mellitus. Table 4 shows the significant variables of the model and the corresponding regression coefficients. Relative weights of the different variables were assigned approximatively according to the Odds ratio to create a simple risk stratification score. Sensitivity and specifity are 0.727 and 0.865 for a cut off value of 3 points, as shown in table 5 . Negative predictive value of a total score $<3$ is 0.842 , positive predictive value is 0.762 for severe disease. AUROC of the score is $0.851(95 \%$ CI $0.745-0.956), \mathrm{p}<0.001$.

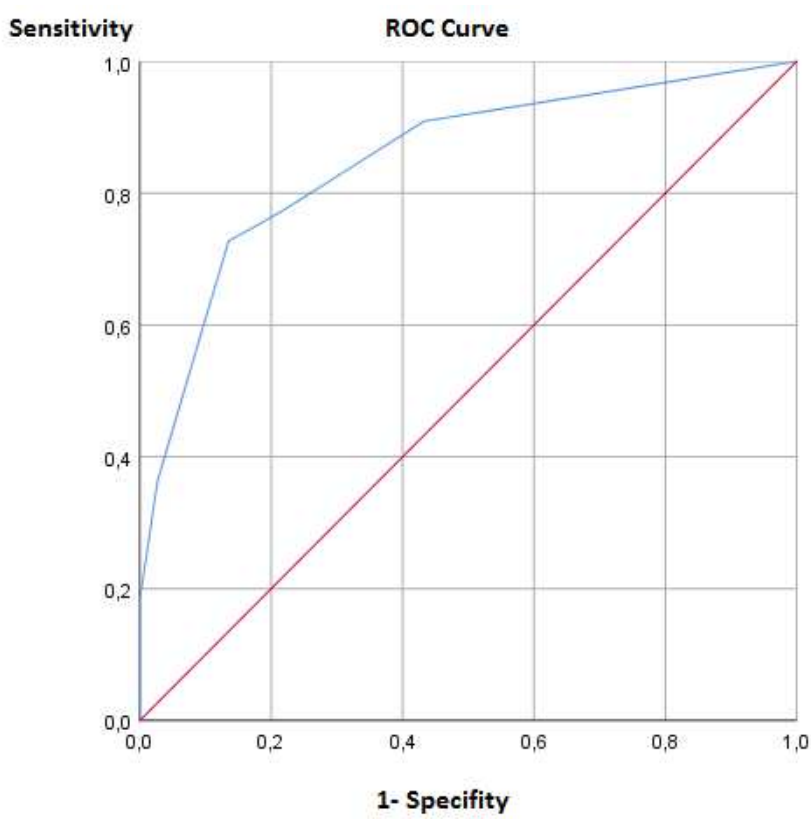

Figure 3. ROC curve of the risk score in the training set. AUROC is 0.851 (95\% CI $0.745-0.956), p<0.001$.

Patients with at least 3 points of the risk-score in the training set are classified as high risk group for severe disease with need for intensive care medicine and/or death within 60 days after admission. The score was evaluated in a second patient cohort (63 patients hospitalized from 11-2020 to 12-2020). Values of sensitivity and specifity of the risk score in the testing set are shown in table 6, corresponding ROC curves in figure 5. Negative predictive value of a score $<3$ is 0.8 ( 0.867 for endpoint "severe disease within five days after admission"), positive predictive value (score $\geq 3$ ) is 0.647 for severe disease (endpoint sever disease overall; 0.588 for endpoint "severe disease within five days after admission"). The Kaplan-Meier survival curve, referring to the initial patient cohort (training set), is shown in figure 5. Log rank test showed significant difference between the groups $(\mathrm{p}=0.001)$.

Table 6. Risk-score, sensitivity and specifity, testing set. a. endpoint "severe disease"

\begin{tabular}{lllll}
\hline Total score & N (63) & Severe disease (\%) & Sensitivity & Specifity \\
\hline 0 & 27 & $4(14.8)$ & 1.0 & 0 \\
1 & 7 & $3(42.9)$ & 0.810 & 0.548 \\
2 & 12 & $3(25)$ & 0.667 & 0.643 \\
3 & 13 & $8(61.5)$ & 0.524 & 0.857 \\
4 & 3 & $2(66.7)$ & 0.143 & 0.976 \\
5 & 1 & $1(100)$ & 0.048 & 1.0 \\
6 & 0 & - & -- & - \\
\hline
\end{tabular}


b. endpoint "severe disease within five days after admission"

\begin{tabular}{lllll}
\hline $\mathbf{0}$ & $\mathbf{2 7}$ & $\mathbf{4}(\mathbf{1 4 . 8 )}$ & $\mathbf{1 . 0}$ & $\mathbf{0}$ \\
\hline 1 & 7 & $3(42.9)$ & 0.824 & 0.522 \\
2 & 12 & $3(25)$ & 0.706 & 0.63 \\
3 & 13 & $8(61.5)$ & 0.588 & 0.848 \\
4 & 3 & $2(66.7)$ & 0.176 & 0.978 \\
5 & 1 & $1(100)$ & 0.059 & 1.0 \\
6 & 0 & - & -- & - \\
\hline
\end{tabular}

In a subgroup analysis of patients, who died, the following

Sensitivity

ROC Curve

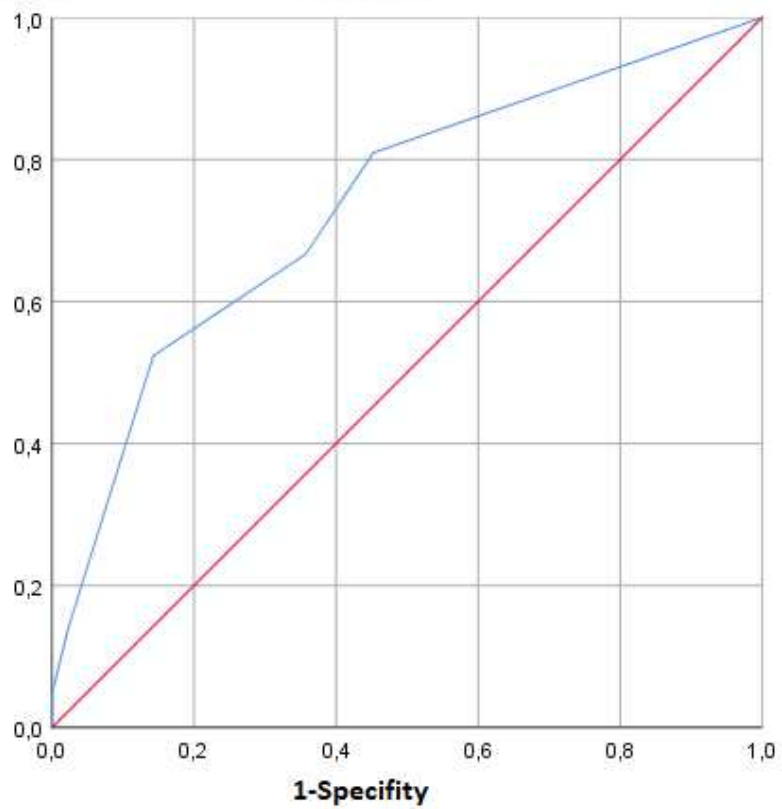

parameters were entered in a cox regression model against the endpoint "death": Age $>65$ years, Diabetes mellitus, elevated Troponin $\mathrm{T}>38 \mathrm{pg} / \mathrm{ml}$, elevated LDH $>460 \mathrm{U} / \mathrm{l}$, chronic kidney disease (stage $\geq 3$ ), serum potassium $\geq 4.7$ $\mathrm{mmol} / \mathrm{l}$. The parameters, shown in table 7, remained in the model. Figures 6 and 7 show the impact on survival of each Troponin $>38 \mathrm{pg} / \mathrm{ml}$ and Diabetes mellitus, figure 8 shows survival of patients with Diabetes mellitus and elevated Troponin $\mathrm{T}>38 \mathrm{pg} / \mathrm{ml}$.

\section{Sensitivity}

ROC Curve

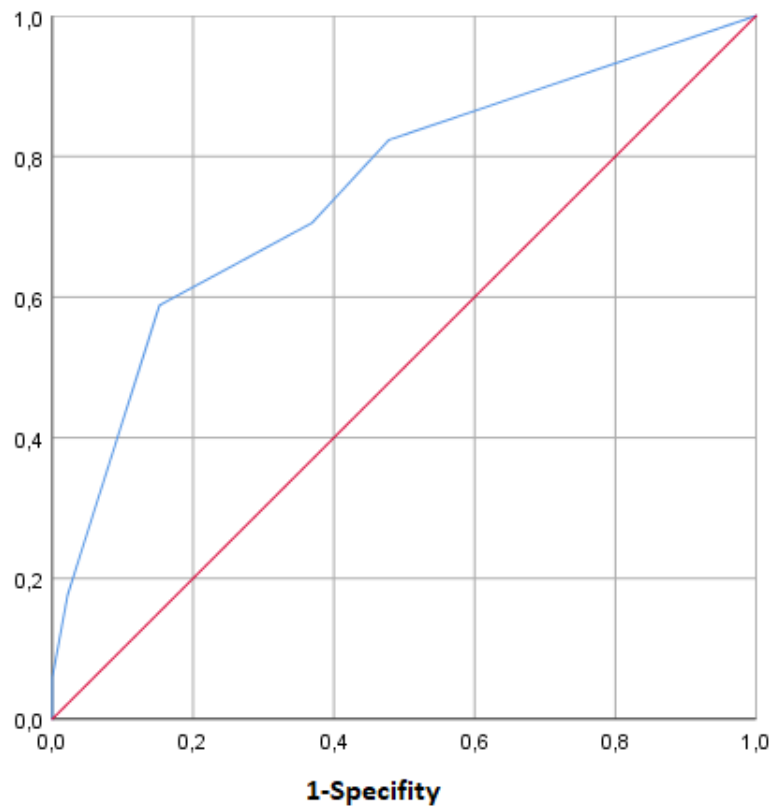

Figure 4. ROC curves of the risk score in the testing set. Left side: characteristic curve for prediction of overall-risk of severe disease (AUROC=0.735, 95\% CI $0.601-0.870, p=0.002)$. Right side: characteristic curve for prediction of risk of severe disease with onset within 5 days after hospital admission $($ AUROC $=0.752,95 \%$ CI $0.609-0.895, p=0.002)$.

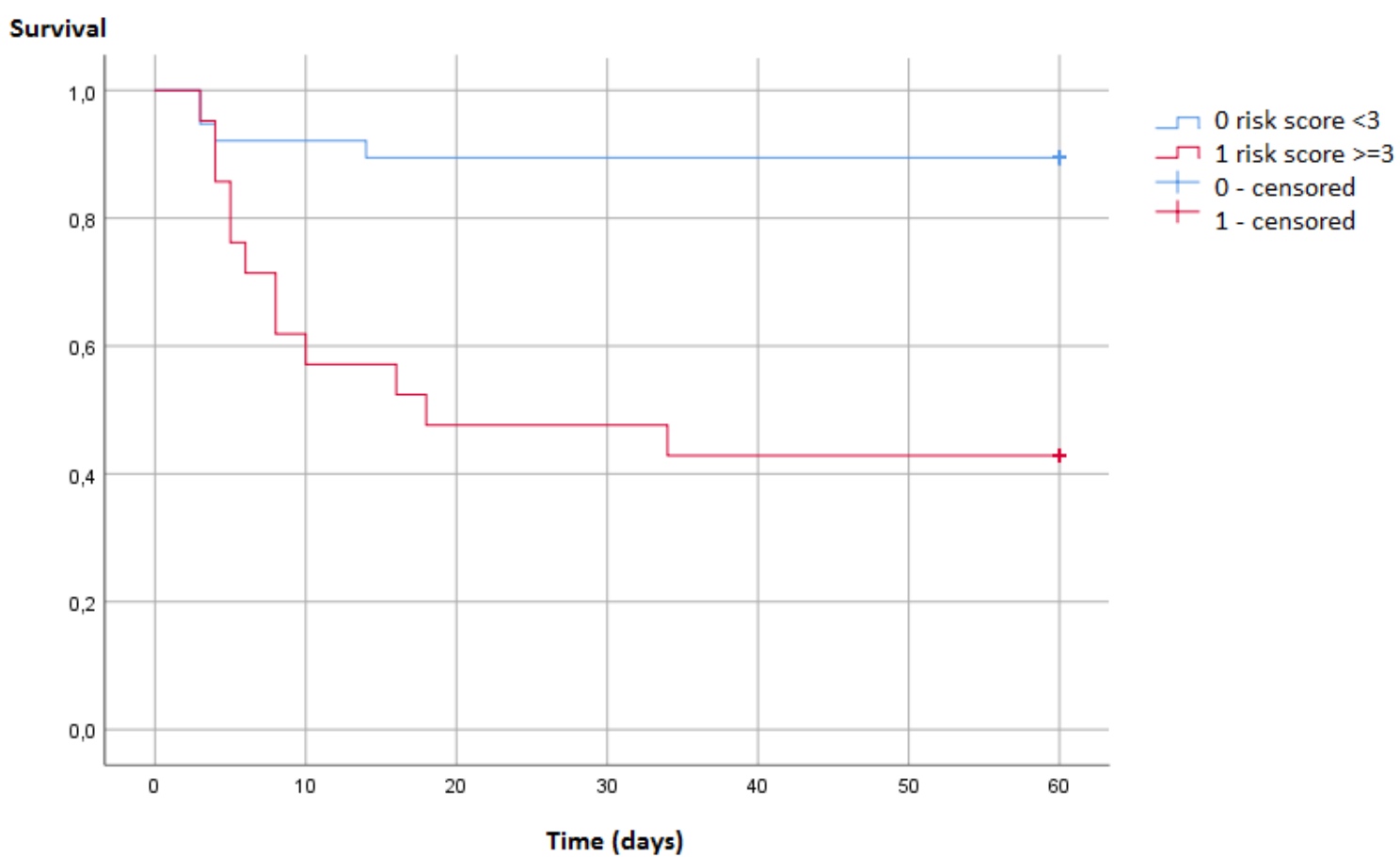

Figure 5. Survival of patients of the training set with at least 3 points of the risk-score compared with patients with low risk (p<0.001). Median survival is 18 days (95\% CI 0-53.89). 
Table 7. Risk factors for death in a Cox-regression model after fourfold stepwise elimination of variables; $p<0.001$.

\begin{tabular}{llll}
\hline p-Value & Hazard & $\mathbf{9 5 \%}$ CI & $2.19-30.88$ \\
\hline Troponin $\mathrm{T}>38 \mathrm{pg} / \mathrm{ml}$ & 0.002 & 8.22 & $1-9.85$ \\
$\mathrm{DM}$ & 0.05 & 3.14 & \\
\hline
\end{tabular}

The bolded values are significant $(\mathrm{p} \leq 0.05)$

\section{Survival}

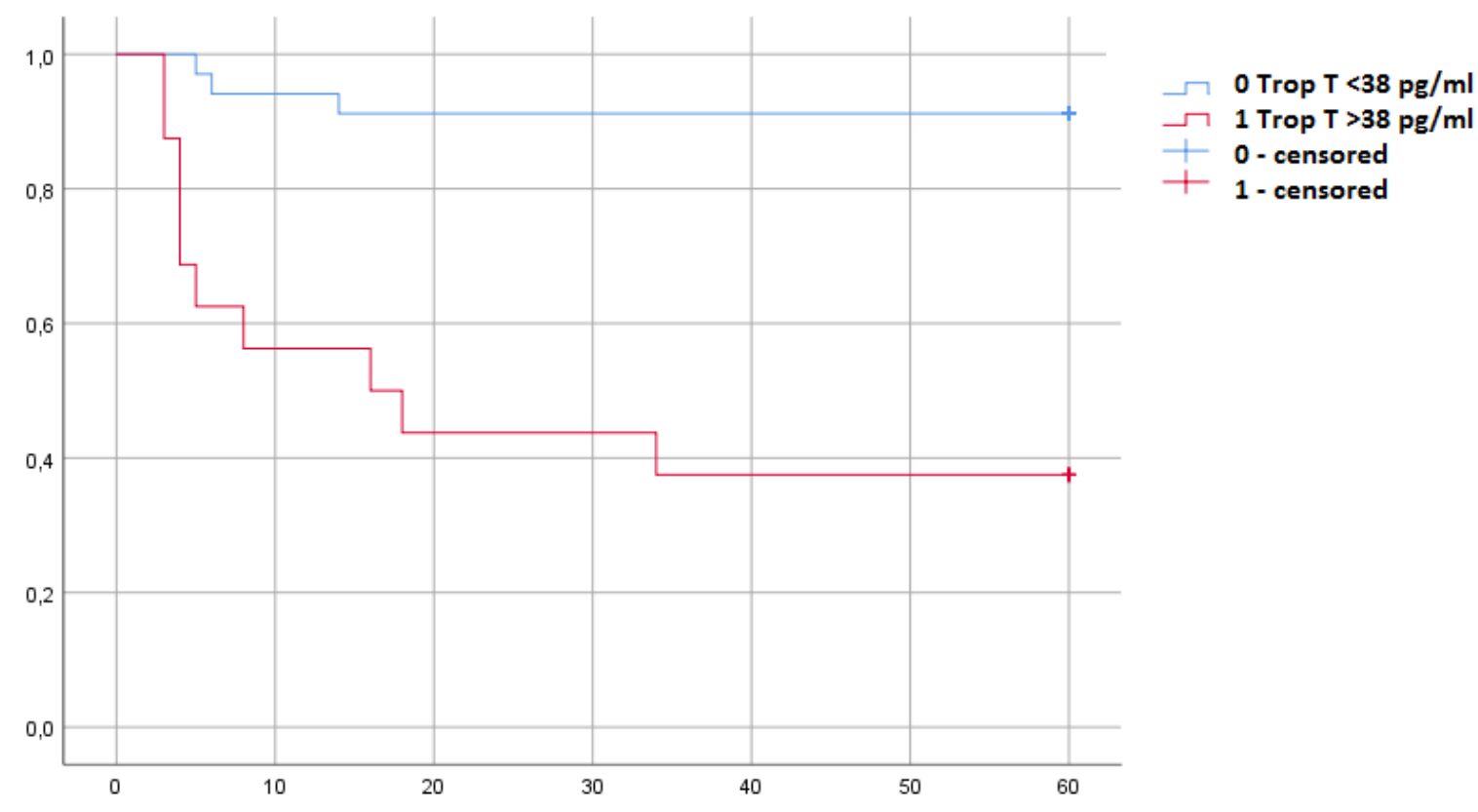

Time (days)

Figure 6. Impact on survival of elevated Troponin T levels $>38 \mathrm{pg} / \mathrm{ml}$. Log rank test: $p<0.001$. Median survival of patients with elevated Troponin T $>38$ $\mathrm{pg} / \mathrm{ml}$ is 16 days $(95 \%$ CI $0-35,6)$.

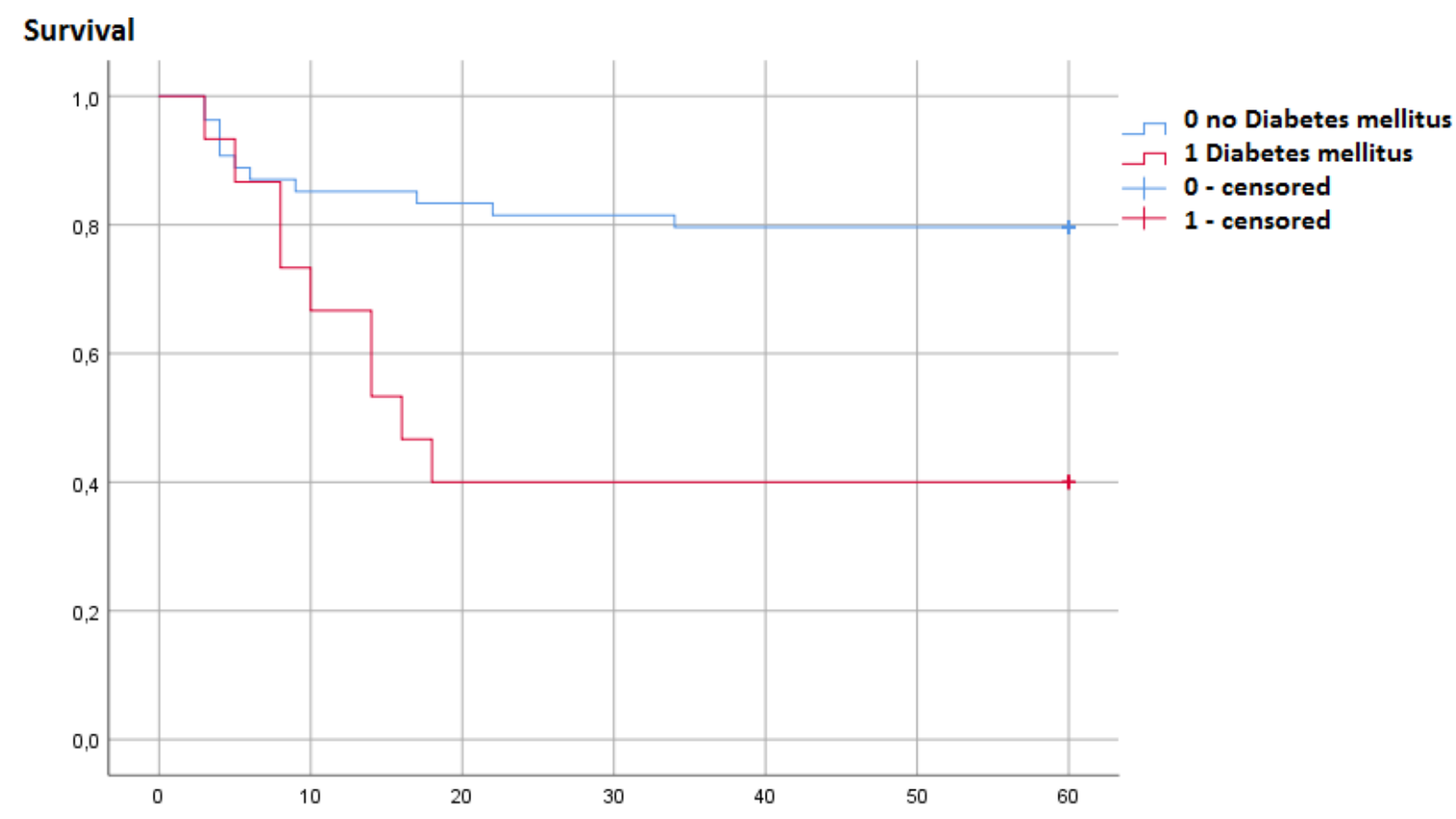

\section{Time (days)}

Figure 7. Impact on survival of Diabetes mellitus as a comorbidity. Median survival of patients with Diabetes mellitus is 16 days (95\% CI 8,43 - 23,57). Log rank test: $p=0.002$. 


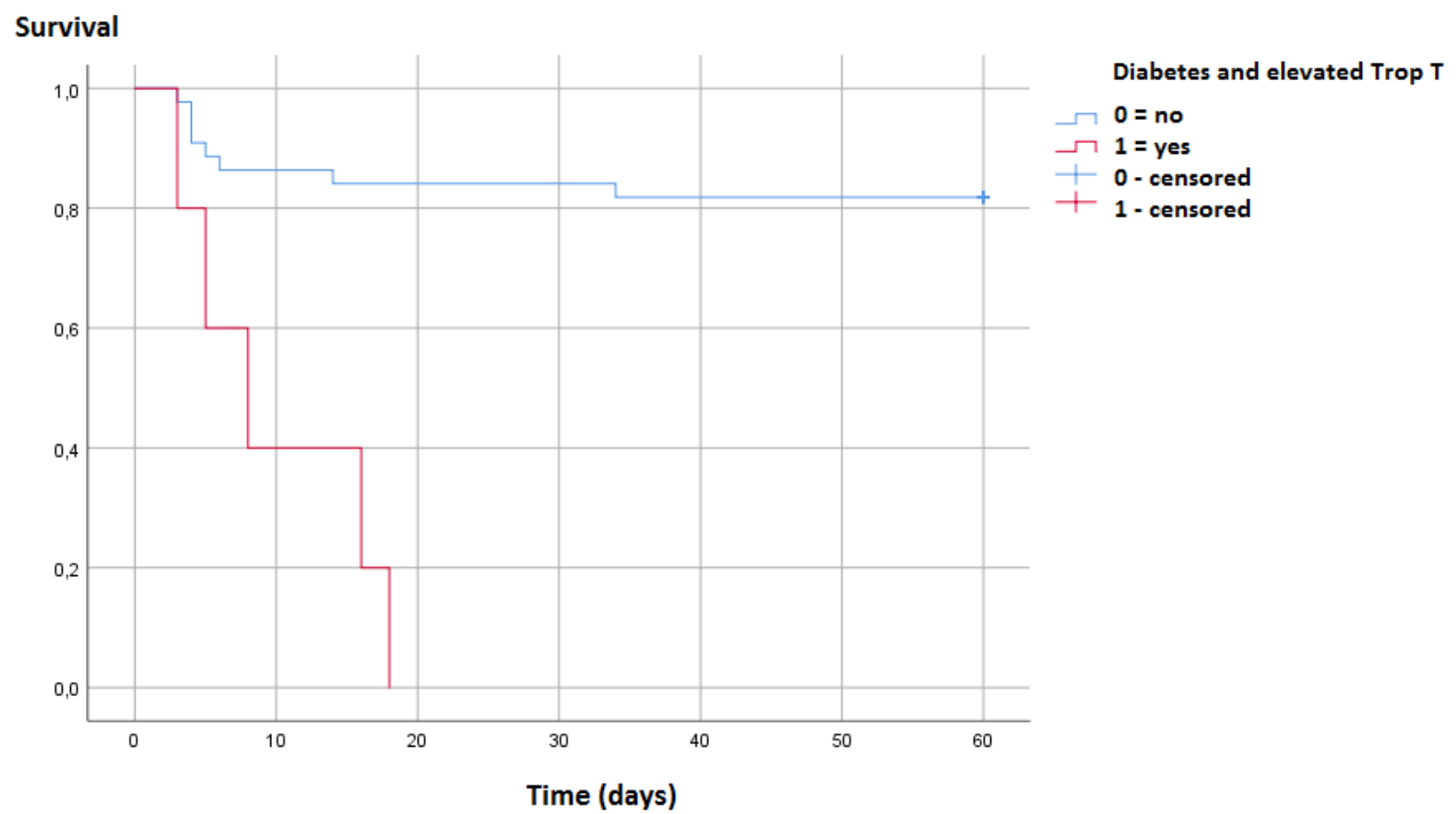

Figure 8. Survival of patients with Diabetes mellitus and elevated Troponin $T>38 \mathrm{pg} / \mathrm{ml}$. Median survival is 8 days (95\% CI 1.56-14.44). Log rank test: $p<0.001$.

\section{Discussion}

In times of increasing incidence of COVID-19, it is important to detect patients with risk for severe illness and to prioritize the limited resources. Most studies of risk factors come from China and results might differ in the western countries. Furthermore, patient cohorts of secondary and tertiary care centers are not directly comparable, as patients with severe illness are in the majority of cases primarily hospitalized in centers with intensive care units providing extracorporal membrane oxygenation. To date, studies from secondary care center do not exist.

In this study, three significant and independent parameters for severe disease were found: elevated Troponin $\mathrm{T}>38$ $\mathrm{ng} / \mathrm{ml}$, elevated LDH $>460 \mathrm{U} / \mathrm{l}$ and Diabetes mellitus. In a review about predicting factors in COVID-19, 60 risk factors were described across 17 publications. Increasing values of age, D-dimer, C-reactive protein, sequential organ failure assessment (SOFA) score, body temperature, decreasing albumin and a history of diabetes were classified as the risk factors with the highest consistency as predictors for COVID-19 severity [7].

Elevated lactate dehydrogenase in serum is referred as a risk factor for severe disease of mid-range consistency [7, 15, 16]. In a study of China, the cut off - value of LDH was 245 $\mathrm{U} / 1$ [15]. With a cut off - value of $460 \mathrm{U} / 1$ following Youden's Index of receiver operator curve, elevated LDH was the parameter with the second highest regression coefficient in our cohort.

Besides age, comorbidities are commonly known as most important risk factors for worse outcome with diabetes being one of the most critical chronic illness [7, 15]. The risk for severe disease in viral infections was already seen in the H1N1 influenza pandemic in 2009, in which patients with diabetes were described as a key susceptibility group for severe H1N1 infections in several studies [17, 18]. There is growing evidence that glycemic fluctuations play an important role in endothelial dysfunction, so glycemic control is very important [19]. Besides immune compromising effects, Diabetes may also deteriorate outcomes in COVID-19 because of increased ACE-2- and Furin- expression, which facilitates entry of coronaviruses into the cells $[18,20]$. Furin is a type-1 membrane-bound protease, member of the proprotein convertase subtilisin/kexin family (PCSK) and is involved in the entry mechanism of corona viruses [21]. Furthermore, IL-6 is increased in patients with diabetes and high IL-6-levels are associated with bad outcome [22]. T-cell function may be impaired in patients with diabetes [23].

Metformin has been described to reduce mortality in diabetics with COVID-19 compared with patients with type 2 diabetes without biguanid therapy in a few observational studies [39-42]. In one study, patients, treated with metformin, had a significantly lower increase in interleukin-6 [41]. Due to its anti-inflammatory activity, Metformin could play a role in patients with diabetes mellitus type 2 and COVID-19, but further investigations and randomized controlled trials are needed to elucidate the effect on prognosis [39, 43].

In our cohort, Diabetes is associated with both severe disease and death, independently. Diabetes is the risk factor with the highest odds ratio for severe disease (17.81 (95\% CI 1.21-263.04)). Taking into account the high prevalence of this chronic disease, it may be the risk factor with the greatest impact on the COVID-19 pandemic, so it is important to take 
care about diabetics with COVID-19 in particular. Only two of fifteen patients with diabetes used metformin before hospital admission, so aforementioned influence on outcome cannot be investigated in our population.

Available data for patients with COPD is contradictory, maybe because of the underrepresentation of these patients in ICU-settings due to their poor prognosis. However, patients with COPD are considered as high risk group and as particularly susceptible to SARS-CoV2-infection with worse prognosis [34].

Interestingly, patients with severe illness tended to an increase of serum sodium level in the course of the disease $(p=0.070)$. This may be the effect of uncontrolled RAASactivation because of down regulation of the ACE2 receptor after endocytosis of the viral complex with consecutive unbraked Angiotensin-II activity, which may contribute to acute lung injury $[24,25]$. As in previous studies described, we see no association of the use of RAAS-inhibitors with death or severe disease [26].

A reduction of ACE2 expression by endothelial cells leads to reduced generation of Ang 1-7 and reduced activation of MAS, which promotes a local pro-thrombotic endothelial cell phenotype. Furthermore, reduced expression of ACE2 might indirectly activate the kallikrein-kinin system (KKS), which leads to increased pro-thrombotic signalling via the activation of bradykinin receptors (BKRs) [27-29]. Prothrombotic condition is a risk factor for myocardial events. Myocardial injury is seen more frequently in patients with COVID-19, who die [30]. Generally cardiac complications are associated with a $60 \%$ increase in pneumonia-associated short-term mortality in community acquired pneumonias [31]. Whether SARS-CoV-2 directly damages myocardial cells after entry via ACE2 or myocardial injury results from inflammatory mediators, is not known [32]. Inflammation plays an important role in acute myocard infarction. In an autopsy study, a significant higher number of inflammatory cells in coronary adventitia was found and this was associated with an increase of incidence of myocardial infarction [33]. Given the pro-thrombotic predisposition in patients with COVID-19, this effect may be aggravated. In accordance with previous findings, elevated levels of high sensitive Troponin is an independent risk factor for death in our cohort [30].

In contrast to other viral infections, some patients develop significant elevation of serum levels of CRP. In autopsy studies, a high number of macrophages was found in lung areas of bronchopneumonia as well as in marginal sinuses of hilar lymph nodes and the marginal zone of the spleen with overexpression of IL-6, suggesting that macrophages may contribute to the overactivation of the immune response leading to a cytokine storm [10-12]. The cytokine storm is seen in patients with severe disease and is associated with poor outcome [13]. CRP is described to increase at the initial stage in patients with severe COVID-19 and is associated with disease development [14]. However, elevated CRP levels were significantly associated with severe illness only in univariate analysis in our study.
As for age, we could see a significant association of older age with death, but there was no association with "severe disease" and, furthermore, age was excluded in multivariate cox regression model because of lack of significance. Age $>65$ years as a predisposing factor for adverse outcome in COVID-19 was described in several studies and meta-analyses and is generally known as a risk factor for severe bacterial or viral pneumonia $[5,7,8,9$, $15,35]$. It may affect the immune system as well as comorbidities.

To date, there is no simple risk stratification tool that allows distinguishing patients with COVID-19 at high respectively low risk in a few minutes. Laboratory parameters, used for a score, should be measured routinely to get the result as quickly as possible. Risk stratification tools like the CURB-65 may not be applicable for patients with COVID-19 because of the special pathophysiology of the disease with wide clinical and immunological spectrum [8]. For assessing the MuLSBTA Score, developed for patients with viral pneumonia, information of bacterial coinfection with positive blood cultures or sputum cultures is needed, but these results do not exist at admission [5]. COVID-GRAM is a risk score with ten items and an AUC in the validation cohort of 0.88 (95\% CI, 0.84-0.93), which was better than the CURB-65, but patient cohorts come from china. Furthermore, the score cannot be used easily in emergency room without internet access in a few minutes [6]. In this study, the risk score consisting of the three identified risk factors for severe disease - elevated LDH $<460 \mathrm{U} / 1$, elevated Troponin $\mathrm{T}>38 \mathrm{pg} / \mathrm{ml}$ and presence of Diabetes mellitus, determined at time of hospital admission - had an acceptable sensitivity $(0.727)$ and specifity $(0.865)$ in the training group, but lacked sensitivity in the validation group $(0.524$, PPV $0.647)$. The specifity was acceptable $(0.857)$ with a negative predicting value of 0.8 . Refering to the combined endpoint (intensive care and/or death) with onset within five days after admission, sensitivity was slightly better $(0.588$, AUC $=0.752$ ). This could be explained by the different stages of disease at time of hospital admission, because respiratory insufficiency and ARDS are described to occur about two weeks after disease onset [37]. However, these significant parameters are available in every emergency room and can be assessed shortly. They may help to identify patients at higher risk for severe disease and conversely can help to classify patients with low risk for death and low probability of need for intensive care medicine albeit the risk cannot be excluded. One should take into account the different stages of disease and the possibility of low values of $\mathrm{LDH}$ and Troponin at initial presentation of the patients, so repetitive testing is recommended.

There are several limitations of this study. First, the case number with 69 patients is low. Analysis of laboratory parameters at admission is performed for only 57 patients because of secondary in-house -infections with SARS-CoV2 in 12 cases. Not all investigated laboratory parameters were available in every patient. Because of the low patient number, not all significant parameters of the univariate association 
analysis of patients with severe disease or death could be included in the regression model. Pulmonary diseases for example were not included in the regression model. Furthermore, adjustment for age is not conducted, given the constricted age distribution and low case number. Secondly, as mentioned above, patients presented with different duration of infection, so laboratory parameters at time of hospital admission may represent different disease stages. Concerning the testing set with 63 patients for the evaluation of the risk score, some patients were treated with dexamethasone unlike the initial patient cohort, because evidence changed in a few months and treatment regimens were adjusted, wherefore both patient collectives are not directly comparable. This could be one of the reasons for the discrepancy of both ROC curves of the risk score in the training and the testing set.

\section{Conclusion}

COVID-19 is a complex infectious disease with variable courses and miscellaneous symptoms. It is important to identify patients at higher risk for severe illness early and to monitor them closely. Conversely, the knowledge of parameters, which indicate a mild disease, is useful to decide, whether in-patients can be admitted to a ward without respirators or not, especially in times of limited capacities of intensive care units. Elevated levels of LDH $(>460 \mathrm{U} / \mathrm{l})$ and/or Troponin $\mathrm{T}(>38 \mathrm{pg} / \mathrm{ml})$ at hospital admission as well as the presence of Diabetes mellitus are relevant parameters with predictive ability in patients with COVID-19, which can help stratifying hospitalized patients into different risk groups. In this connection, diabetics with elevated levels of Troponin $\mathrm{T}$ should be considered as high risk group. Risk stratification tools should be simply and quickly applicable in emergency rooms. Therefore, the use of routine laboratory parameters, as mentioned above, is reasonable. Further studies with greater number of patients are needed to develop a risk stratification tool with high sensitivity and specifity in predicting the outcome of patients with COVID-19, so that therapies (e.g. with dexamethasone) start earlier and prognosis gets better.

\section{References}

[1] Xiaochen Li, Shuyun $\mathrm{Xu}$, Muqing Yu, Risk factors for severity and mortality in adult COVID-19 inpatients in Wuhan, J Allergy Clin Immunol. 2020 Jul; 146 (1): 110-118, doi: 10.1016/j.jaci.2020.04.006. Epub 2020 Apr 12

[2] Zainab Shahid, BS, Ricci Kalayanamitra, BS, Brendan McClafferty, COVID -19 and Older Adults: What We Know, J Am Geriatr Soc. 2020 May, doi: 10.1111/jgs. 16472

[3] WHO-China Joint Mission. Report of the WHO-China Joint Mission on Coronavirus Disease 2019 (COVID-19). https://www.who.int/docs/default-source/coronaviruse/whochina-joint-mission-on-covid-19-final-report.pdf. Accessed March 26, 2020.
[4] Uranga, A., Quintana, J. M., Aguirre, U., Predicting 1-year mortality after hospitalization for community-aquired pneumonia, PloS One 13: e0192750, doi: 10.1371/journal.phone.0192750

[5] Lingxi Guo, Dong Wei, Xinxin Zhang, Clinical Features Predicting Mortality Risk in Patients With Viral Pneumonia: The MuLBSTA Score, Front. Microbiol. 10: 2752, doi: 10.3389/fmicb.2019.02752

[6] Wenhua Liang, Hengrui Liang, Limin Ou, Development and Validation of a Clinical Risk Score to Predict the Occurrence of Critical Illness in Hospitalized Patients With COVID-19, JAMA Intern Med. 2020 Aug; 180 (8): 1-9. doi: 10.1001/jamainternmed.2020.2033

[7] Rod J. E., Oviedo-Trespalacios Oscar, Cortes-Ramirez Javier, A brief-review of the risk factors for covid-19 severity, Rev Saude Publica. 2020; 54: 60, doi: 10.11606/s15188787.2020054002481

[8] García Luis F., Immune Response, Inflammation, and the Clinical Spectrum of COVID-19, Front Immunol. 2020; 11: 1441, doi: 10.3389/fimmu.2020.01441

[9] Almirall J., Serra-Prat M., Bolíbar I., Risk Factors for Community-Acquired Pneumonia in Adults: A Systematic Review of Observational Studies, Respiration 2017; 94: 299311, doi: $10.1159 / 000479089$

[10] Paces J, Strizova Z, Smrz D, COVID-19 and the immune system, Physiol Res Actions. 2020 Jul 16; 69 (3): 379-388. doi: $10.33549 /$ physiolres. 934492 .

[11] Barton L. M., Duval E. J., Stroberg E., COVID-19 Autopsies, Oklahoma, USA, Am J Clin Pathol. 2020 May 5; 153 (6): 725-733. doi: 10.1093/ajcp/aqaa062.

[12] Park M. D., Macrophages: a Trojan horse in COVID-19? Nat Rev Immunol. 2020 Apr 17: 1. doi: 10.1038/s41577-0200317-2 [Epub ahead of print]

[13] Huang C., Wang Y., Li X., Clinical features of patients infected with 2019 novel coronavirus in Wuhan, China, Lancet, 2020 Feb 15; 395 (10223): 497-506. doi: 10.1016/S0140-6736(20)30183-5. Epub 2020 Jan 24.

[14] Tan C., Huang Y., Shi F., C-reactive protein correlates with computed tomographic findings and predicts severe COVID19 early, J Med Virol. 2020 Apr 25: 10.1002/jmv.25871., doi: $10.1002 / j m v .25871$

[15] Zhou F., Yu T., Du R. Clinical course and risk factors for mortality of adult inpatients with COVID-19 in Wuhan, China: a retrospective cohort study, Lancet. 202028 March-3 April; 395 (10229): 1054-1062, doi: 10.1016/S01406736(20)30566-3

[16] Ponti G., Maccaferri M., Ruini C., Biomarkers associated with COVID-19 disease progression, Crit Rev Clin Lab Sci. 2020: 1-11, doi: 10.1080/10408363.2020.1770685

[17] Hulme K. D., Gallo L. A., Short K. R., Influenza Virus and Glycemic Variability in Diabetes: A Killer Combination? Front Microbiol. 2017; 8: 861, doi: 10.3389/fmicb.2017.00861

[18] Singh AK, Gupta R, Ghosh A, Misra A., Diabetes in COVID19: Prevalence, pathophysiology, prognosis and practical considerations, Diabetes Metab Syndr. Jul-Aug 2020; 14 (4): 303-310, doi: 10.1016/j.dsx.2020.04.004. 
[19] Ceriello A., Esposito K., Piconi L., Oscillating glucose is more deleterious to endothelial function and oxidative stress than mean glucose in normal and type 2 diabetic patients. Diabetes. 2008 May; 57 (5): 1349-54, DOI: 10.2337/db08-0063.

[20] Rao S., Lau A., and So H-C. Exploring diseases/traits and blood proteins causally related to 284 expression of ACE2, the putative receptor of 2019-nCov: a Mendelian Randomization analysis Highlights Tentative Relevance of Diabetes-Related Traits. vol. 285, Diabetes Care. 2020 Jul; 43 (7): 1416-1426. doi: $10.2337 / \mathrm{dc} 20-0643$.

[21] Fernandez C., Rysa J., Almgren P., Plasma levels of the proprotein convertase furin and incidence of diabetes and mortality. J Intern Med. 2018; 284: 377-387, doi: 10.1111 joim. 12783

[22] Maddaloni E., Buzzetti R. Covid-19 and diabetes mellitus: unveiling the interaction of two pandemics. Diabetes Metab Res Rev. 2020 Mar 31 doi: 10.1002/dmrr.3321

[23] Kulcsar K. A., Coleman C. M., Beck S. E., Frieman M. B. Comorbid diabetes results in immune dysregulation and enhanced disease severity following MERS-CoV infection. JCI Insight. 2019; 4 (20): 131774. doi: 10.1172/jci.insight.131774.pii.

[24] Vaduganathan M., Vardeny O., Michel T., Renin-AngiotensinAldosterone-System Inhibitors in Patients with Covid-19, Engl J Med. 2020 Mar 30: NEJMsr2005760. doi: 10.1056/NEJMsr2005760

[25] Yang P, Gu H, Zhao Z, et al. Angiotensin-converting enzyme 2 (ACE2) mediates influenza H7N9 virus-induced acute lung injury. Sci Rep 2014; 4: 7027-7027, doi: 10.1038/srep07027

[26] Reynolds H. R., Samrachana Adhikari S., Pulgarin C., ReninAngiotensin-Aldosterone System Inhibitors and Risk of Covid-19, N Engl J Med. 2020 May 1: NEJMoa2008975, doi: 10.1056/NEJMoa2008975

[27] Perico L, Benigni A., Casiraghi F., Immunity, endothelial injury and complement-induced coagulopathy in COVID-19, Nat Rev Nephrol. 2020 Oct 19: 1-19. doi: 10.1038/s41581020-00357-4

[28] Teuwen L. A., Geldhof V., Pasut A., COVID-19: the vasculature unleashed, Nat Rev Immunol. 2020 Jul; 20 (7): 389-391, doi: 10.1038/s41577-020-0343-0.

[29] Garvin MR, Alvarez C, Miller JI, A mechanistic model and therapeutic interventions for COVID-19 involving a RASmediated bradykinin storm, Elife. 2020 Jul 7; 9: e59177. doi: 10.7554/eLife.59177

[30] Shi S., Qin M., Cai Y., Characteristics and clinical significance of myocardial injury in patients with severe coronavirus disease 2019, Eur Heart J. 2020 May 11: ehaa408., doi: 10.1093/eurheartj/ehaa408

[31] Corrales-Medina VF, Musher DM, Wells GA, Cardiac complications in patients with community-acquired pneumonia: incidence, timing, risk factors, and association with short-term mortality. Circulation. 2012 Feb 14; 125 (6): 773-81. doi: 10.1161/CIRCULATIONAHA.111.040766.

[32] Oudit G. Y., Kassiri Z., Jiang C., SARS-coronavirus modulation of myocardial ACE2 expression and inflammation in patients with SARS, Eur J Clin Invest. 2009 Jul; 39 (7): 618-25., doi: 10.1111/j.1365-2362.2009.02153.x. Epub 2009 May 6.

[33] Madjid M., Vela D., Khalili-Tabrizi H., Systemic infections cause exaggerated local inflammation in atherosclerotic coronary arteries: clues to the triggering effect of acute infections on acute coronary syndromes, Tex Heart Inst J. 2007; 34 (1): 11-8.,

[34] Olloquequi J., COVID-19 Susceptibility in chronic obstructive pulmonary disease, Eur J Clin Invest. 2020 Oct; 50 (10): e13382. doi: 10.1111/eci.13382.

[35] Zhaohai Zheng Z., Peng F., Xu B., Risk factors of critical \& mortal COVID-19 cases: A systematic literature review and meta-analysis, J Infect. 2020 Aug; 81 (2): e16-e25. Doi: 10.1016/j.jinf.2020.04.021.

[36] Sardu C., Gambardella J., Morelli M. B., Hypertension, Thrombosis, Kidney Failure, and Diabetes: Is COVID-19 an Endothelial Disease? A Comprehensive Evaluation of Clinical and Basic Evidence, J Clin Med. 2020 May; 9 (5): 1417. doi: $10.3390 / \mathrm{jcm} 9051417$

[37] Yang X, Yu Y, Xu J, Clinical course and outcomes of critically ill patients with SARS-CoV-2 pneumonia in Wuhan, China: a single-centered, retrospective, observational study. The Lancet Respiratory medicine. 2020; 8 (5): 475-81

[38] Scheen A. J., Metformin and COVID-19: From cellular mechanisms to reduced mortality, Diabetes Metab. 2020 Nov; 46 (6): 423-426, doi: 10.1016/j.diabet.2020.07.006

[39] Bramante C., Ingraham N., Murray T. et al., Observational study of metformin and risk of mortality in patients hospitalized with Covid-19. medRxiv. 2020; 28 doi: $0.1101 / 2020.06 .19 .20135095$

[40] Cariou B., Hadjadj S., Wargny M. et al., Phenotypic characteristics and prognosis of in-patients with COVID-19 and diabetes: the CORONADO study. Diabetologia. 2020; 63: 1500-1515, doi: 10.1007/s00125-020-05180-x.

[41] Chen Y., Yang D., Cheng B. et al., Clinical characteristics and outcomes of patients with diabetes and COVID-19 in association with glucose-lowering medication. Diabetes Care. 2020; 43: 1399-1407, doi: 10.2337/dc20-0660.

[42] Luo P., Qiu L., Liu Y. et al., Metformin treatment was associated with decreased mortality in COVID-19 patients with diabetes in a retrospective analysis. Am J Trop Med Hyg. 2020; 103: 69-72, doi: 10.4269/ajtmh.20-0375.

[43] Cameron A. R., Morrison V. L., Levin D. et al., AntiInflammatory Effects of Metformin Irrespective of Diabetes Status, Circ Res Actions. 2016 Aug 19; 119 (5): 652-65. doi: 10.1161/CIRCRESAHA.116.308445. 